\title{
复杂异形曲面自动建模图像矩视觉伺服定位控制"
}

\author{
彭伟星 $^{1,2}$ 王耀南 ${ }^{1,2}$ 曾 凯 ${ }^{1,2}$ 吴昊天 ${ }^{1,2}$ \\ (1. 湖南大学电气与信息工程学院 长沙 410082;
}

2. 湖南大学机器人视觉感知与控制技术国家工程实验室 长沙 410082)

\begin{abstract}
摘要: 异形曲面目标的表面深度信息无法进行参数化表示, 是难以建立矩特征交互矩阵的主要原因。提出一种利用目标轮廓 深度信息直接构建矩特征交互矩阵的新方法, 推导出传统图像矩交互矩阵的非参数化形式。考虑到传统矩特征视觉伺服定位 过程的响应速度慢、收玫范围小, 设计一种新型的深度矩作为视觉伺服的图像特征, 并推导出其非参数化交互矩阵。采用最 速下降法设计视觉伺服控制器, 分析并比较两种矩特征视觉伺服控制方法在异形曲面定位过程的动态性能。结果表明, 非参 数化形式的传统矩交互矩阵和深度矩交互矩阵均有效, 两种控制方法在初始偏差较小时是渐近稳定的。提出的深度矩视觉伺 服方法与传统的矩特征视觉伺服相比, 较为明显地改善了收玫速度和收玫范围。
\end{abstract}

关键词: 复杂异形曲面; 自动建模; 图像矩视觉伺服; 定位控制

中图分类号: TP13

\section{Positioning of Autonomous Modeling Manipulator for Deformed Surface Using Moments-based Visual Servoing}

\author{
PENG Weixing $^{1,2}$ WANG Yaonan ${ }^{1,2}$ ZENG Kai $^{1,2}$ WU Haotian ${ }^{1,2}$
}

(1. College of Electrical and Information Engineering, Hunan University, Changsha 410082;

2. National Engineering Laboratory for Robot Visual Perception and Control Technology,

Hunan University, Changsha 410082)

\begin{abstract}
The surface depth information of the deformed surface target cannot be parameterized, which is the main reason why it is difficult to establish the image interaction matrix of moment feature. Therefore, a new method for constructing the nonparametric interaction matrix of the traditional image moment is derived based on the target contour depth information. Considering that the traditional moment based visual servo control has slow response speed and small convergence range, a novel depth moment is designed as the visual servo image feature, and its non-parametric interaction matrix is derived. The visual servo controller is designed using the steepest descent method, and dynamic performance of the two moment feature based visual servo control methods in the process of profiled surface positioning is analyzed and compared. The results show that nonparametric form of both the traditional moment interaction matrix and the depth moment interaction matrix are effective. Both methods are asymptotically stable in the control process. Compared with the traditional moment feature based visual servo method, the depth moment based visual servo improves the convergence speed and convergence range.
\end{abstract}

Key words: deformed surface; autonomous modeling; moments-based visual servoing; positioning

\section{0 前言}

航空航天、海洋工程、新能源装备等高端装备 领域是国家的战略性行业。在这些领域广泛存在着 航空发动机叶片、舰船螺旋桨叶片、水轮机叶片等 复杂异形曲面零部件。

以航空发动机叶片为例, 由于叶片空气动力学

* 国家自然科学基金资助项目(61733004, 61573134, 61433016)。20181207 收到初稿, 20190507 收到修改稿
特性的要求，叶片必须具有精确的尺寸、准确的形 状和严格的表面完整性 ${ }^{[1]}$ 。但是其壁薄、扭曲大、 型面复杂, 容易产生形变, 严重影响加工精度和表 面质量。利用精密测量和检测技术指导叶片加工过 程是控制叶片加工误差、保证型面精度的关键 ${ }^{[2]}$ 。

传统方式采用手工测量工具(游标卡尺、千分尺 等)测量零件尺寸和形状, 效率低、误差较大 ${ }^{[3]}$ 。三 坐标测量机通过分析采样点的坐标测得对象参数, 是一种应用最广泛的自动化测量设备, 也是目前精 度最高的测量方法 ${ }^{[4]}$ 。但该方法属于接触式测量, 
可能造成薄壁叶片形变, 同时精度容易受测量路 径 ${ }^{[5]}$ 、采样点数量 ${ }^{[6]}$ 和测量头补偿方法的影响 ${ }^{[7]}$ 。近 年来, 激光扫描技术作为一种非接触式测量方法逐 渐兴起, 它通过获取对象表面的大量点云表示采样 结果, 测量精度较高, 适应能力强 ${ }^{[8]}$ 。然而, 手持式 扫描仪的工作效率和数据完整性严重依赖人工的熟 练度 ${ }^{[9]}$ 。利用灵活的多自由度机器人替代人工, 携 带非接触式传感器获取三维数据是一种提高测量系 统性能的新技术。

如图 1 所示, 对未知模型物体进行自主建模的 核心思想是用三维扫描仪多次扫描物体,增量式建 成物体表面完整模型 ${ }^{[10]}$ 。机器人自动建模系统分成 三个功能模块 ${ }^{[10]}$ : 模型更新(Model update)、扫描规 划(Scan planning)、位姿跟踪(Pose tracking)。

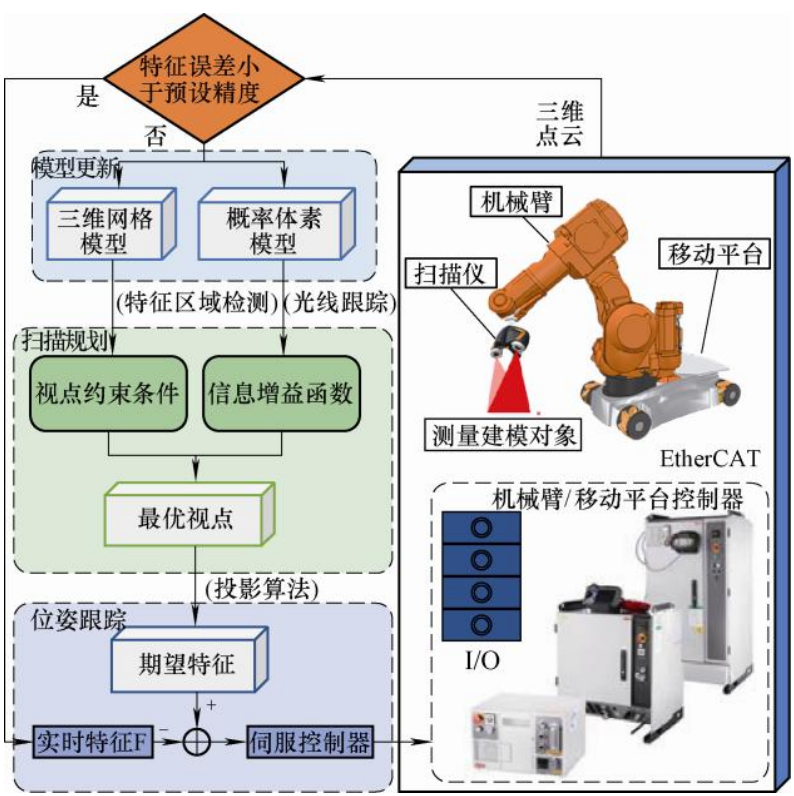

图 1 机器人测量系统

模型更新阶段将每一次扫描获得的局部点云融 合到已建成的模型, 点云的配准精度最终决定了三 维模型的精度。同时, 生成的部分三角网格模型 (Triangular mesh)和体素模型 (Probabilistic voxel space, PVS)可用于扫描规划。

扫描规划在自动建模过程中属于下一最优视点 问题(Next-best-viewpoint, NBV), 最优视点指在约 束条件下，一个能获得最多目标未知信息的三维传 感器位姿 ${ }^{[11]}$ 。最优视点规划一般有两个阶段 ${ }^{[12]}$ : 第 一阶段在三维网格模型的边界和孔洞上(特征区域) 计算候选位姿; 第二阶段通过光线跟踪(Ray tracing) 技术在 PVS 上计算信息增益( $(\mathrm{IG}), \mathrm{IG}$ 最大的位姿即 为最优位姿。扫描规划决定模型的完整性。

位姿跟踪指对扫描规划获得的最优传感器位姿 进行定位。定位误差是无法完全规避的, 其存在会
严重降低建模精度、采样密度等指标。目前, 多数 研究专注于对建模目标的高覆盖率扫描 ${ }^{[13]}$, 而较少 研究如何直接提高扫描位姿定位精度。为了提高建 模精度, 通常利用测量载体(机械臂、飞行器)的定 位系统进行粗定位, 再对获取的点云进行精确配准 以提高建模精度。这种定位方法相对建模目标属于 一种开环定位方法, 缺乏对环境的抗扰动能力; 且 该方法要对移动平台进行室内定位, 装载在机器人 末端的三维传感器需要手眼标定, 最终的定位精度 受到室内定位精度、手眼标定精度和机器人绝对定 位精度三个方面的影响, 难以满足点云精确配准的 需求。

基于图像的视觉伺服(IBVS)具有一定的环境适 应能力, 而且正以图像空间的特征作为误差对相机 位姿进行闭环控制, 所以对手眼标定和移动平台的 定位精度具有良好的鲁棒性。因此, IBVS 在自动测 量系统中具有较好的应用前景。同时，投影算法可 以将 NBV 转换成期望图像特征, 为 IBVS 应用于自 动测量中提供了一定可行性。

相当一部分视觉伺服以平面目标为研究对象, 用视觉伺服方法定位曲面目标需要考虑选取与目标 深度信息存在联系的图像特征, 以及如何构建图像 特征的交互矩阵。

最成熟的基于特征点坐标的视觉伺服, 优点是 交互矩阵求解简单。但在实用性方面，该方法存在 文献[14]描述的收玫性、稳定性问题，且对特征点 匹配精度要求非常高。

文献[15-16]提出的基于全局图像灰度的视觉伺 服和文献[17]提出的基于深度地图(Depth map)的视 觉伺服在深度变化剧烈的复杂场景中可以实现定 位, 但是交互矩阵庞大, 需要建立一个 $M \times N$ 行 6 列的交互矩阵 $(M \times N$ 为图像尺寸), 特征维数 $k=M \times N>6$ ，无法保证全局稳定性 ${ }^{[18]}$ 。

图像矩属于统计学特征, 实际上包含了图像的 全局信息。文献[19-21]提出的基于图像矩的视觉伺 服相较于上述方法具有交互矩阵尺寸小和控制解耦 性良好的优势, 且图像矩的变化与目标深度变化相 关，满足定位曲面目标的第一个前提。然而，无法 参数化表示曲面表面的深度信息, 将导致难以建立 图像矩的交互矩阵。

\section{1 改进的图像矩视觉伺服}

\section{1 传统图像矩视觉伺服定位曲面目标的局限性 传统图像矩定义为 ${ }^{[19]}$}




$$
m_{i j}(t)=\iint_{S} x^{i} y^{j} \mathrm{~d} x \mathrm{~d} y
$$

式中, $(i+j)$ 表示图像矩的阶数, $S$ 为图像 $\boldsymbol{\pi}$ 中的 目标区域。

在求图像矩的交互矩阵前, 首先将目标区域的 深度函数建立成高维多项式, 目标为曲面时

$$
\frac{1}{Z}=\sum_{k \geqslant 0, j \geqslant 0} A_{k j} x^{k} y^{j} \quad(x, y) \in S
$$

式中, $Z$ 表示图像中某一点 $(x, y)$ 的深度, $A_{k j}$ 为多 项式中 $(k+j)$ 次项的系数, 相机与目标的相对位姿 不变时是实常数。求得深度函数的解析式后, 用函 数参数表示的图像矩交互矩阵为

$$
\boldsymbol{L}_{m_{i j}}^{\mathrm{T}}=\left(\begin{array}{c}
-\sum_{p, q}(i+p) A_{p q} m_{i+p-1, j+q} \\
-\sum_{p, q}(j+q) A_{p q} m_{i+p, j+q-1} \\
-\sum_{p, q}(i+j+p+q+2) A_{p q} m_{i+p, j+q} \\
(i+j+3) m_{i, j+1}+j m_{i, j-1} \\
-(i+j+3) m_{i+1, j}+i m_{i-1, j} \\
i m_{i-1, j+1}-j m_{i+1, j-10}
\end{array}\right)
$$

目标为平面时, 其深度函数模型可以表示成一 维线性函数

$$
\frac{1}{Z}=A x+B y+Z_{g} \quad(x, y) \in S
$$

式中, $Z_{g}$ 为目标区域中心的深度, $A 、 B$ 在相机与 目标的相对位姿不变时为实常数。此时, 深度矩交 互矩阵的解析形式为

$$
\boldsymbol{L}_{m_{i j}}^{\mathrm{T}}=\left(\begin{array}{c}
-i\left(A m_{i j}+B m_{i-1, j+1}+C m_{i-1, j}\right)-A m_{i, j} \\
-j\left(A m_{i+1, j-1}+B m_{i j}+C m_{i, j-1}\right)-B m_{i, j} \\
(i+j+3)\left(A m_{i+1, j}+B m_{i, j+1}+C m_{i, j}\right)-C m_{i, j} \\
(i+j+3) m_{i, j+1}+j m_{i, j-1} \\
-(i+j+3) m_{i+1, j}+i m_{i-1, j} \\
i m_{i-1, j+1}-j m_{i+1, j-1}
\end{array}\right)
$$

用文献[19]中提出的上述方法求交互矩阵时, 首先要求建立图像中目标区域的深度函数。利用深 度函数参数求交互矩阵存在如下问题。

(1) 控制精度方面: 深度函数的准确性直接影 响交互矩阵的正确性, 进一步影响控制精度。当目 标为不规则的曲面时, 将深度函数拟合成式(2)要求 的形式本身是一个复杂的过程。

(2) 控制实时性方面: 在相机移动过程中深度 函数是时变的, 为了保证控制精度, 需要实时建立 深度函数。但是, 控制过程中的每次迭代都进行深 度函数建模将导致算法复杂度增加。

\section{2 基于轮廓深度的直接交互矩阵}

根据第 1.1 节的描述，用传统图像矩视觉伺服 定位曲面目标时的局限性在于难以求得交互矩阵, 而求解交互矩阵的难点在于必须实时建立目标区域 的深度函数。针对该问题，本文推导了一种无需深 度函数参数的交互矩阵求解方法。

相机的运动过程 中, 相机速度 $\boldsymbol{V}=\left(v_{x}, v_{y}, \boldsymbol{w}_{z}, \omega_{x}, \boldsymbol{a}_{\mathrm{o}}\right.$ 根据文献[19]，图像矩导 数与目标区域中像素点的关系为

$$
\dot{m}_{i j}=\iint_{S}\left[\frac{\partial f}{\partial x} \dot{x}+\frac{\partial f}{\partial y} \dot{y}+f(x, y)\left(\frac{\partial \dot{x}}{\partial x}+\frac{\partial \dot{y}}{\partial y}\right)\right] \mathrm{d} x \mathrm{~d} y
$$

为了避免用深度函数的参数求交互矩阵，本文 假设图像中每个像素点的深度信息 $Z$ 已知, 并直接 代入到式(6)求交互矩阵。图像 $\boldsymbol{\pi}$ 中任意一点 $\boldsymbol{X}=(x, y)$ 与相机速度之间的线性关系为 $\left\{\begin{array}{l}\dot{x}=-1 / Z \cdot v_{x}+x / Z \cdot v_{z}+x y \cdot \omega_{x}-\left(1+x^{2}\right) \cdot \omega_{y}+y \cdot \omega_{z} \\ \dot{y}=-1 / Z \cdot v_{y}+y / Z \cdot v_{z}+\left(1+y^{2}\right) \cdot \omega_{x}-x y \cdot \omega_{y}-x \cdot \omega_{z}\end{array}\right.$

对式(7)求导得

$$
\left\{\begin{array}{l}
\frac{\partial \dot{x}}{\partial x}=\frac{\partial-\frac{1}{z}}{\partial x} v_{x}+0+\frac{\partial \frac{x}{z}}{\partial x} v_{z}+y \omega_{x}-2 x \omega_{y}+0 \\
\frac{\partial \dot{y}}{\partial y}=0+\frac{\partial-\frac{1}{z}}{\partial y} v_{y}+\frac{\partial \frac{y}{z}}{\partial y} v_{z}+2 y \omega_{x}-x \omega_{y}+0
\end{array}\right.
$$

将式(7)、(8)代入式(6), 推导出图像矩交互矩阵为

$$
\boldsymbol{L}_{m_{i j}}^{\mathrm{T}}=\left(\begin{array}{c}
\iint_{S}\left(-\frac{1}{Z} i x^{i-1} y^{j}+\frac{\partial \frac{-1}{Z}}{\partial x} x^{i} y^{j}\right) \mathrm{d} x \mathrm{~d} y \\
\iint_{S}\left(-\frac{1}{Z} j x^{i} y^{j-1}+\frac{\partial \frac{-1}{Z}}{\partial y} x^{i} y^{j}\right) \mathrm{d} x \mathrm{~d} y \\
\iint_{S}\left[\frac{i}{Z} x^{i} y^{j}+\frac{j}{Z} x^{i} y^{j}+\left(\frac{\partial \frac{x}{Z}}{\partial x}+\frac{\partial \frac{y}{Z}}{\partial y}\right) x^{i} y^{j}\right] \mathrm{d} x \mathrm{~d} y \\
(i+j+3) m_{i, j+1}+j m_{i, j-1} \\
-(i+j+3) m_{i+1, j}-i m_{i-1, j} \\
i m_{i-1, j+1}-j m_{i+1, j-1}
\end{array}\right.
$$

利用格林理论, 将式(9)简化成关于轮廓深度信 息的直接交互矩阵的形式, 即

$$
\boldsymbol{L}_{m_{i j}}^{\mathrm{T}}=\left(\begin{array}{c}
\oint_{C} \frac{-1}{Z} x^{i} y^{j} \mathrm{~d} y \\
\oint_{C} \frac{-1}{Z} x^{i} y^{j} \mathrm{~d} x \\
\oint_{C} \frac{x^{i+1} y^{j}}{Z} \mathrm{~d} y+\oint_{C} \frac{x^{i} y^{j+1}}{Z} \mathrm{~d} x \\
(i+j+3) m_{i, j+1}+j m_{i, j-1} \\
-(i+j+3) m_{i+1, j}-i m_{i-1, j} \\
i m_{i-1, j+1}-j m_{i+1, j-1}
\end{array}\right)
$$


式中, $\boldsymbol{C}$ 表示目标区域的轮廓。

式(10)表示的图像矩交互矩阵不再依赖于深度 函数的参数, 通过轮廓 $\boldsymbol{C}$ 上的深度信息可直接求得 图像矩交互矩阵。在实际应用过程中, 需要实时获 取目标区域轮廓上的深度信息, 现有的三维传感器 可满足此要求。

\section{2 基于深度矩的视觉伺服方法}

第 1 节改进的图像矩视觉伺服方法实际上在偏 差较大的情况下是无法收玫的。针对该问题, 本文 提出一种的新的深度矩视觉伺服方法, 本节重点讨 论深度矩交互矩阵的推导过程。

\section{1 深度矩定义}

图像 $\boldsymbol{\pi}$ 中目标区域 $\boldsymbol{S}$ 的深度矩定义如下

$$
m_{p q}=\iint_{S} x^{q} y^{q} Z((x, y), t) \mathrm{d} x \mathrm{~d} y
$$

式中, $(p+q)$ 表示图像矩阶数, $Z((x, y), t)$ 为图像 的深度函数。

\section{2 深度矩的直接交互矩阵}

从深度矩 $m_{p q}$ 的导数 $\dot{m}_{p q}$ 出发, 推导深度矩交互 矩阵。当相机位姿发生变化时, 区域 $\boldsymbol{S}$ 内的深度信 息和轮廓 $\boldsymbol{C}$ 的位置均随之发生变化, 推导 $\dot{m}_{p q}$ 是一 个复杂的过程。

如图 $2 \mathrm{~b}$ 所示, $\boldsymbol{A}$ 为相邻两个时刻目标区域的 重叠部分, $\boldsymbol{B}$ 表示非重叠部分。 $\dot{m}_{p q}$ 实际上等于 $\boldsymbol{A}$ 和 $\boldsymbol{B}$ 内的深度矩导数之和, 即

$$
\dot{m}_{p q}=\dot{m}_{p q}(\boldsymbol{A})+\dot{m}_{p q}(\boldsymbol{B})
$$

式中, $\dot{m}_{p q}(\boldsymbol{A})$ 表示区域 $\boldsymbol{A}$ 内深度矩的导数, $\dot{m}_{p q}(\boldsymbol{B})$ 表示区域 $\boldsymbol{B}$ 内深度矩的导数。

$\dot{m}_{p q}(\boldsymbol{A})$ 仅与在区域 $\boldsymbol{A}$ 中的深度信息的变化有 关，所以

$$
\begin{gathered}
\dot{m}_{p q}(\boldsymbol{A})=\iint_{A} x^{q} y^{q} \dot{Z}((x, y), t) \mathrm{d} x \mathrm{~d} y \approx \\
\iint_{S} x^{q} y^{q} \dot{Z}((x, y), t) \mathrm{d} x \mathrm{~d} y
\end{gathered}
$$

图像中任意某个点的深度变化与相机速度之间 的关系为

$$
\dot{Z}=\boldsymbol{L}_{Z} \boldsymbol{V}
$$

式中, $\boldsymbol{L}_{Z}$ 为深度交互矩阵, 由此可得

$$
\dot{m}_{p q}(\boldsymbol{A})=\iint_{S} x^{q} y^{q} \boldsymbol{L}_{Z} \mathrm{~d} x \mathrm{~d} y \boldsymbol{V}
$$

即

$$
\boldsymbol{L}_{m_{p q}}(\boldsymbol{A})=\iint_{S} x^{q} y^{q} \boldsymbol{L}_{Z} \mathrm{~d} x \mathrm{~d} y
$$

式中， $\boldsymbol{L}_{m_{p q}}(\boldsymbol{A})$ 表示区域 $\boldsymbol{A}$ 内的深度矩交互矩阵。 根据文献[17]

$$
\boldsymbol{L}_{Z}=\left[\frac{\alpha}{Z} \frac{\beta}{Z}-\frac{Z+\alpha x+\beta y}{Z} Z_{\omega_{x}} Z_{\omega_{y}} Z_{\omega_{z}}\right]
$$

式中, $\alpha=\frac{\partial Z}{\partial x}, \beta=\frac{\partial Z}{\partial y}, Z_{\omega_{x}}=-y Z-x y \alpha+\left(1+y^{2}\right) \beta$, $Z_{\omega_{y}}=x Z+\left(1+x^{2}\right) \alpha-x y \beta, \quad Z_{\omega_{z}}=x \beta-y \alpha$ 。

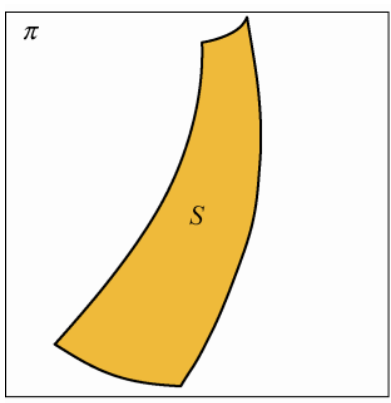

(a) 目标图像

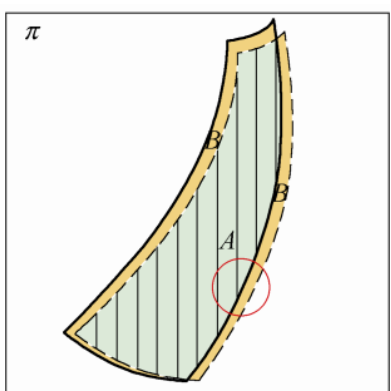

(b) 重叠区域划分示意图

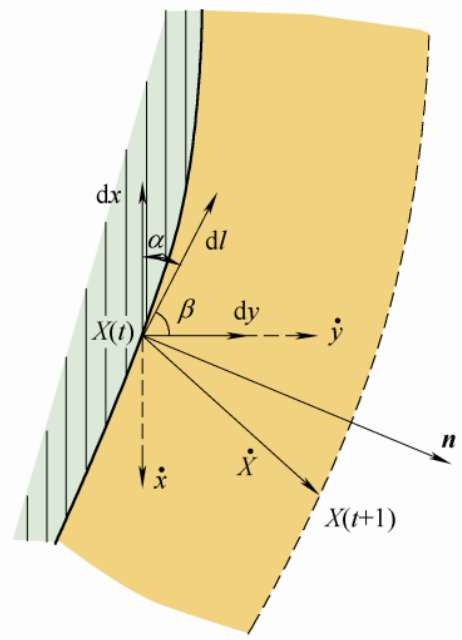

(c) 轮廓微分量示意图

图 2 目标图像、重叠区域划分示意图和轮廓微分量示意图 因此，可推导出区域 $\boldsymbol{A}$ 内的深度矩交互矩阵

$$
\boldsymbol{L}_{m_{p q}}^{\mathrm{T}}(\boldsymbol{A})=\left(\begin{array}{c}
\iint_{S} x^{q} y^{q} \frac{\alpha}{Z} \mathrm{~d} x \mathrm{~d} y \\
\iint_{S} x^{q} y^{q} \frac{\beta}{Z} \mathrm{~d} x \mathrm{~d} y \\
\iint_{S} x^{q} y^{q}\left(-\frac{Z+\alpha x+\beta y}{Z}\right) \mathrm{d} x \mathrm{~d} y \\
\iint_{S} x^{q} y^{q} \mathrm{Z}_{\omega_{x}} \mathrm{~d} x \mathrm{~d} y \\
\iint_{S} x^{q} y^{q} \mathrm{Z}_{\omega_{y}} \mathrm{~d} x \mathrm{~d} y \\
\iint_{S} x^{q} y^{q} \mathrm{Z}_{\omega_{z}} \mathrm{~d} x \mathrm{~d} y
\end{array}\right.
$$

式中, $\dot{m}_{p q}(\boldsymbol{B})$ 表示相机变化过程中, 目标轮廓在图 
像中位置变化导致的深度矩变化。如图 $2 \mathrm{c}$ 所示, $\dot{X}$ 表示轮廓上一点 $\boldsymbol{X}$ 的速率, 可将 $\dot{\boldsymbol{X}}$ 分解成 $\boldsymbol{x}$ 方向和 $\boldsymbol{y}$ 方向的的速率, 即 $\dot{\boldsymbol{X}}=\dot{\boldsymbol{x}}+\dot{\boldsymbol{y}} ; \mathrm{d} \boldsymbol{l}$ 为轮廓的微分 量, 可以对其进行分解, 即 $\mathrm{d} \boldsymbol{l}=\mathrm{d} \boldsymbol{x}+\mathrm{d} \boldsymbol{y}, \alpha 、 \beta$ 分 别表示 $\mathrm{d} \boldsymbol{l}$ 与 $\mathrm{d} \boldsymbol{x} 、 \mathrm{~d} \boldsymbol{y}$ 的夹角; $\boldsymbol{n}$ 为 $\mathrm{d} \boldsymbol{l}$ 的单位法矢 量。因此

$$
\begin{gathered}
\dot{m}_{p q}(\boldsymbol{B})=\oint_{C(t)} x^{q} y^{q} Z((x, y), t) \dot{\boldsymbol{X}} \boldsymbol{n} \mathrm{d} l= \\
\iint_{S}\left[\frac{\partial x^{q} y^{q} Z}{\partial x} \dot{x}+\frac{\partial x^{q} y^{q} Z}{\partial y} \dot{y}+x^{q} y^{q} Z\left(\frac{\partial \dot{x}}{\partial x}+\frac{\partial \dot{y}}{\partial y}\right)\right] \mathrm{d} x \mathrm{~d} y
\end{gathered}
$$

经推导, 得到区域 $\boldsymbol{B}$ 内的深度矩交互矩阵 $\boldsymbol{L}_{m_{p q}}(\boldsymbol{B})$ 为

$$
\boldsymbol{L}_{m_{p q}}^{\mathrm{T}}(\boldsymbol{B})=\left(\begin{array}{c}
\oint_{C} x^{p} y^{q} \mathrm{~d} y \\
\oint_{C} x^{p} y^{q} \mathrm{~d} x \\
\oint_{C} x^{p+1} y^{q} \mathrm{~d} y+\oint_{C} x^{p} y^{q+1} \mathrm{~d} x \\
{\left[(p+q+3) m_{p, q+1}+q m_{p, q-1}+\right.} \\
\left.\iint_{S} \frac{\partial Z}{\partial x} x^{p+1} y^{q+1}+\frac{\partial Z}{\partial y}\left(x^{p} y^{q}+x^{p} y^{q+2}\right) \mathrm{d} x \mathrm{~d} y\right] \\
{\left[-(p+q+3) m_{p+1, q}+p m_{p-1, q}-\right.} \\
\left.\iint_{S} \frac{\partial Z}{\partial x}\left(x^{p} y^{q}+x^{p+2} y^{q}\right)+\frac{\partial Z}{\partial y} x^{p+1} y^{q+1} \mathrm{~d} x \mathrm{~d} y\right] \\
{\left[p m_{p-1, q+1}-q m_{p+1, q-1}+\right.} \\
\left.\iint_{S} \frac{\partial Z}{\partial x} x^{p} y^{q+1}-\iint_{S} \frac{\partial Z}{\partial y} x^{p+1} y^{q} \mathrm{~d} x \mathrm{~d} y\right]
\end{array}\right)
$$

根据文献[22]中提出的简化方法

$$
\begin{aligned}
& \iint_{S} \frac{\partial Z}{\partial x} x^{p} y^{q} \mathrm{~d} x \mathrm{~d} y=-p m_{p-1, q}+\oint_{C} x^{p} y^{q} Z \mathrm{~d} y \\
& \iint_{S} \frac{\partial Z}{\partial y} x^{p} y^{q} \mathrm{~d} x \mathrm{~d} y=-q m_{p, q-1}+\oint_{C} x^{p} y^{q} Z \mathrm{~d} x
\end{aligned}
$$

代入式(20), 最终得到 $\boldsymbol{L}_{m_{p q}}(\boldsymbol{B})$ 的简化形式

$$
\boldsymbol{L}_{m_{p q}}^{\mathrm{T}}(\boldsymbol{B})=\left(\begin{array}{c}
\oint_{C} x^{p} y^{q} \mathrm{~d} y \\
\oint_{C} x^{p} y^{q} \mathrm{~d} x \\
\oint_{C} x^{p+1} y^{q} \mathrm{~d} y+\oint_{C} x^{p} y^{q+1} \mathrm{~d} x \\
\oint_{C}\left(x^{p} y^{q}+x^{p} y^{q+2}\right) Z \mathrm{~d} x+\oint_{C} x^{p+1} y^{q+1} Z \mathrm{~d} y- \\
\oint_{C} x^{p+1} y^{q+1} Z \mathrm{~d} x-\oint_{C}\left(x^{p} y^{q}+x^{p+2} y^{q}\right) Z \mathrm{~d} y- \\
\oint_{C} x^{p+1} y^{q} Z \mathrm{~d} x+\oint_{C} x^{p} y^{q+1} Z \mathrm{~d} y
\end{array}\right)
$$

实际上, 传统图像矩与深度信息只是间接相关, 而所提出的新型的深度矩直接引入了目标的深度信 息与目标区域内像素的统计信息, 其变化对深度更 加敏感。

\section{3 控制策略}

\section{1 特征 $\boldsymbol{F}$ 选择}

本文选取 $\boldsymbol{F}=\left(x_{g}, y_{g}, a, p_{x}, p_{y}, \alpha\right)$, 构成特征矢 量的六个量由各阶图像矩构成

$$
\begin{gathered}
x_{g}=\frac{m_{10}}{m_{00}} \quad y_{g}=\frac{m_{01}}{m_{00}} \quad a=m_{00} \\
\alpha=\frac{1}{2} \arctan \left(\frac{2 \mu_{11}}{\mu_{20}-\mu_{02}}\right)
\end{gathered}
$$

式中, $\mu_{p q}$ 表示中心矩, $p_{x}$ 与 $p_{y}$ 为两个不变矩

$$
\begin{aligned}
& p_{x}=\left(c_{2} c_{3}+s_{2} c_{3}\right) / k \\
& p_{y}=\left(s_{2} c_{3}+c_{2} s_{3}\right) / k
\end{aligned}
$$

式中, $c_{3}=c_{1}^{2}-s_{1}^{2}, s_{3}=2 s_{1} c_{1}, k=I_{1} I_{3}^{\frac{3}{2}} / \sqrt{a}$ 。

$$
\left\{\begin{array}{l}
I_{1}=c_{1}^{2}+s_{1}^{2} I_{2}=c_{2}^{2}+s_{2}^{2} \quad I_{3}=\mu_{20}+\mu_{02} \\
c_{1}=\mu_{20}-\mu_{02} \quad s_{1}=2 \mu_{11} \\
c_{2}=\mu_{03}-3 \mu_{21} \quad s_{2}=\mu_{30}-3 \mu_{12}
\end{array}\right.
$$

特征对应的交互矩阵求解方法可详细参考文献[19]。

\section{2 控制器设计}

本文仍采用传统的控制律

$$
\boldsymbol{V}=-\lambda \boldsymbol{L}_{F d}^{-1}\left(\boldsymbol{F}-\boldsymbol{F}_{d}\right)
$$

式中, $\lambda$ 为一个适当大小的增益, $\boldsymbol{L}_{F d}$ 为相机处于 位姿时求得的图像交互矩阵。

\section{4 试验结果和分析}

\section{1 仿真平台}

为了验证改进的图像矩交互矩阵的求解方法, 和提出的新型深度矩视觉伺服方法的有效性, 本文 建立了如图 3 所示的仿真平台，对具有曲面特性的 叶片模型进行了定位试验。

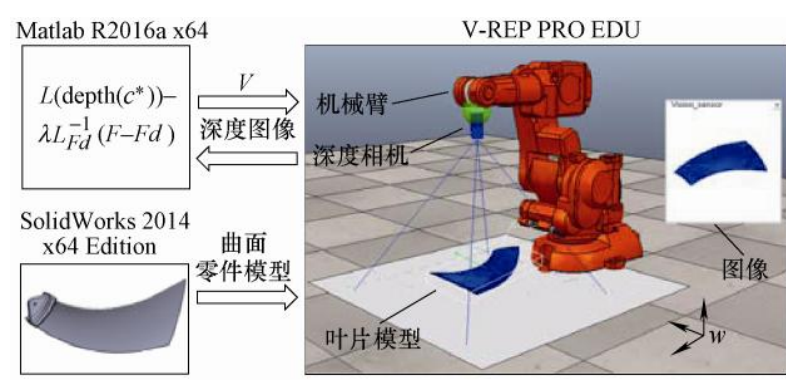

图 3 仿真平台

(1) V-REP PRO EDU 物理仿真平台提供一台 6 自由度机器人机械臂、一台分辨率 $256 \times 256$ 的深度 
相机，相机与机械臂之间为 eye-in-hand 配置。

(2) 由 SolidWorks 2014 x64 提供图 4 所示的叶 片模型。

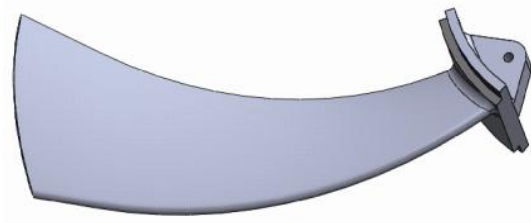

图 4 叶片模型

(3) Matlab R2016 x64 平台用于图像处理、计算 视觉伺服控制器输出。图像处理过程的目的是实时 计算特征 $\boldsymbol{F}$ 以及获取目标轮廓 $\mathbf{C}$ 的坐标点集用于 计算交互矩阵。

\section{2 仿真结果及分析}

首先在相机初始位姿偏差为 $(0.14 \mathrm{~m}, 0.11 \mathrm{~m}$, $\left.-0.5 \mathrm{~m}, 10^{\circ}, 10^{\circ}, 20^{\circ}\right)$ 时进行了曲面的定位试 验。图 5 所示为传统图像矩视觉伺服的试验结果, 图像矩交互矩阵通过 3.2 节提出的直接法求得; 图 6 所示为深度矩视觉伺服的试验结果。收敛时相机的 位姿误差见表 1 。

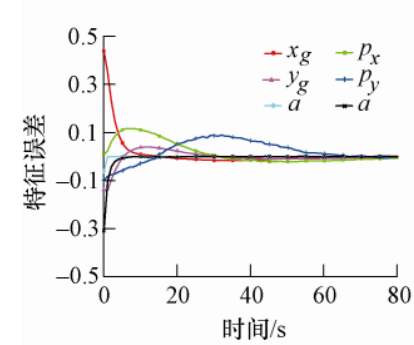

(a) 特征误差

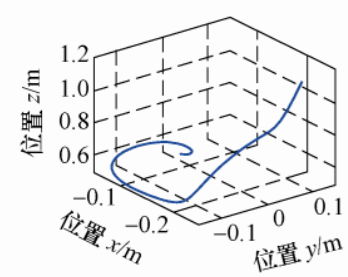

(c) 相机空间轨迹

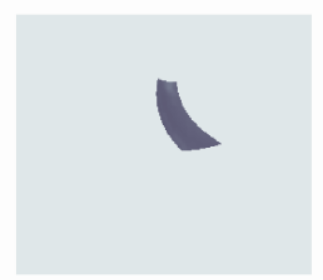

(e) 初始图像 (b) 相机6自由度速度

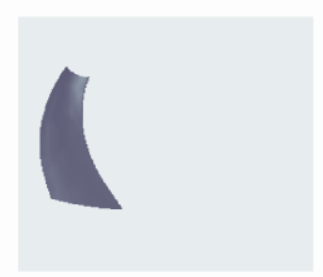

(d) 期望图像 I $^{*}$

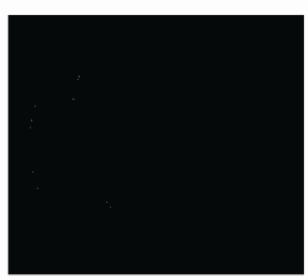

(f) 稳态时 $I-I^{*}$

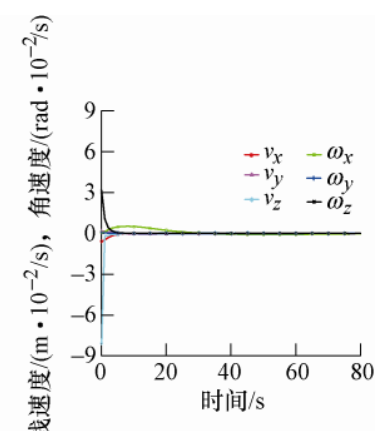

图 5 初始位姿偏差为 $\left(0.14 \mathrm{~m}, 0.11 \mathrm{~m},-0.5 \mathrm{~m}, 10^{\circ}\right.$, $\left.10^{\circ}, 20^{\circ}\right)$ 的传统图像矩视觉伺服定位试验

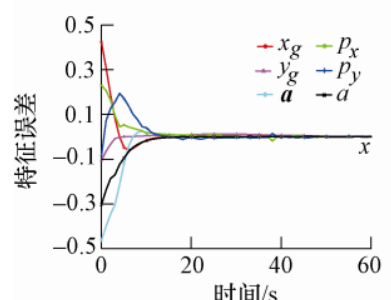

(a) 特征误差

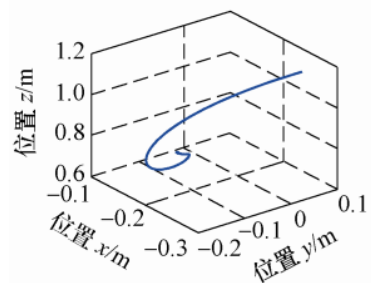

(c) 相机空间轨迹

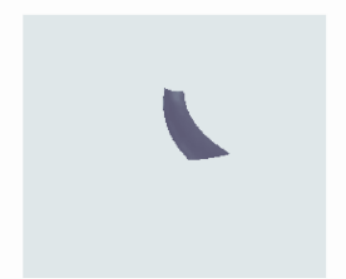

(e) 初始图像

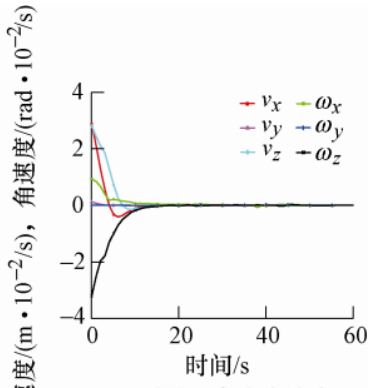

(b) 相机6自由度速度

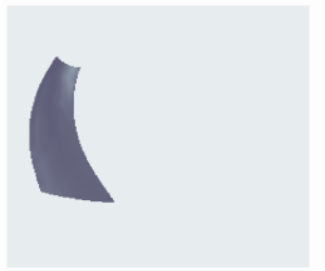

(d) 期望图像 I*

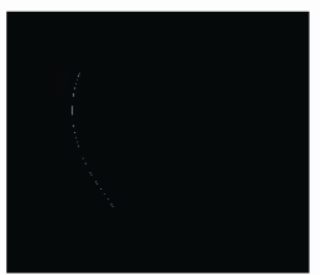

(f) 稳态时 $I-I^{*}$
图 6 初始位姿偏差为 $\left(0.14 \mathrm{~m}, 0.11 \mathrm{~m},-0.5 \mathrm{~m}, 10^{\circ}\right.$, $\left.10^{\circ}, 20^{\circ}\right)$ 的深度矩视觉伺服定位试验

表 1 初始偏差较小的定位稳态误差

\begin{tabular}{ccccccc}
\hline & \multicolumn{5}{c}{ 位姿误差绝对值 } \\
\cline { 2 - 5 } \cline { 5 - 7 } & $\Delta_{x} / \mathrm{mm}$ & $\Delta_{y} / \mathrm{mm}$ & $\Delta_{z} / \mathrm{mm}$ & $\theta_{x} /\left(^{\circ}\right)$ & $\theta_{y} /\left(^{\circ}\right)$ & $\theta_{z} /\left(^{\circ}\right)$ \\
\hline $\begin{array}{c}\text { 传统矩 } \\
\text { 视觉伺服 }\end{array}$ & 2.02 & 1.27 & 1.91 & 0.10 & 0.23 & 0.08 \\
$\begin{array}{c}\text { 深度矩 } \\
\text { 视觉伺服 }\end{array}$ & 2.04 & 2.08 & 1.73 & 0.21 & 0.16 & 0.02 \\
\hline
\end{tabular}

图 5a、6a 所示的特征变化趋势以及稳态时相 机的位姿误差表明两种方法均可实现曲面的定位, 即证明本文理论部分所推导的两种矩特征交互矩 阵是正确的。两者的定位精度相差较小，但传统图 像矩视觉伺服在 $t=70 \mathrm{~s}$ 附近才到达稳态, 深度矩视 觉伺服仅在 $t=20 \mathrm{~s}$ 附近已经收玫, 说明定位过程中 深度矩视觉伺服在收敛速度方面明显优于传统图 像矩视觉伺服。同时对比图 5c、6c，深度矩视觉伺 服定位过程相机的空间轨迹优于传统图像矩视觉 伺服。

表 1 中, 深度矩视觉伺服方法在 $x 、 y$ 和 $\theta_{x}$ 三 个自由度的定位误差比传统方法大。为了验证深度 矩视觉伺服方法是否在改善收敛速度的同时增大定 位误差, 在相同条件下从 $(0.07 \mathrm{~m}, 0.06 \mathrm{~m},-0.25 \mathrm{~m}$, $\left.5^{\circ}, 5^{\circ}, 15^{\circ}\right) \sim\left(0.21 \mathrm{~m}, 0.18 \mathrm{~m},-0.7 \mathrm{~m}, 12^{\circ}\right.$, 
$\left.12^{\circ}, 22^{\circ}\right)$ 范围内增加了 19 组初始偏差, 并进行 定位试验。计算 20 组定位试验收敛时 6 个自由度的 位姿误差绝对值的平均值, 见表 2 。

表 2 初始偏差较小的 20 组试验稳态误差平均值

\begin{tabular}{ccccccc}
\hline & \multicolumn{6}{c}{ 位姿误差绝对值 } \\
\cline { 2 - 3 } \cline { 5 - 6 } & $\Delta_{x} / \mathrm{mm}$ & $\Delta_{y} / \mathrm{mm}$ & $\Delta_{z} / \mathrm{mm}$ & $\theta_{x} /\left(^{\circ}\right)$ & $\theta_{y} /\left(^{\circ}\right)$ & $\theta_{z} /\left(^{\circ}\right)$ \\
\hline $\begin{array}{c}\text { 传统矩 } \\
\text { 视觉伺服 }\end{array}$ & 2.32 & 1.14 & 2.27 & 0.22 & 0.21 & 0.11 \\
$\begin{array}{c}\text { 深度矩 } \\
\text { 视觉伺服 }\end{array}$ & 2.21 & 2.33 & 1.87 & 0.35 & 0.15 & 0.09 \\
\hline
\end{tabular}

深度矩视觉伺服方法在 $y$ 和 $\theta_{x}$ 两个自由度上 定位误差比传统方法稍大, 在另外四个自由度上的 误差较小。该方法主要提高了收玫速度, 定位精度 没有较大的改善。

在同样的试验条件下, 扩大相机的初始位姿偏 差至 $\left(0.52 \mathrm{~m}, 0.33 \mathrm{~m},-1.5 \mathrm{~m}, 15^{\circ}, 15^{\circ}, 25^{\circ}\right)$ 。 图 7 所示为传统图像矩视觉伺服的定位结果, 图像 矩交互矩阵通过第 3.2 节提出的直接法求得; 图 8 所示为深度矩视觉伺服的定位结果。收敛时位姿误 差见表 3。

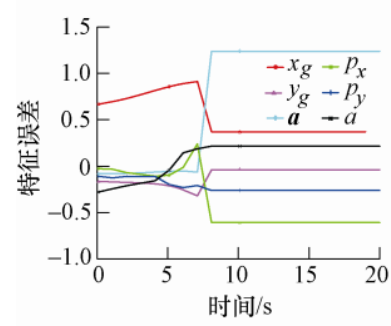

(a) 特征误差

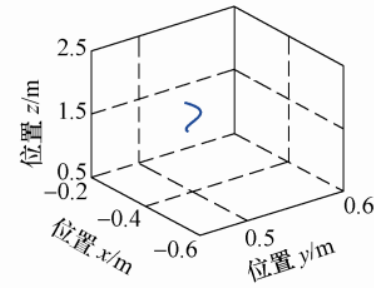

(c) 相机空间轨迹

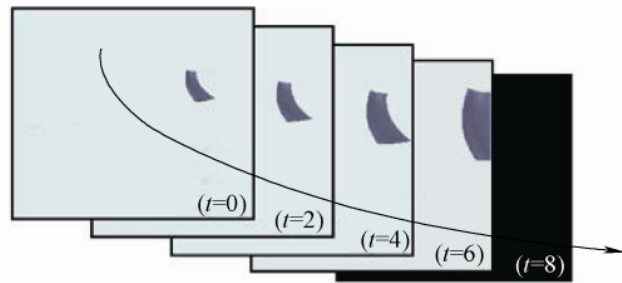

(e) $t=0 、 t=2 、 t=4 、 t=6 、 t=8$ 时刻图像

图 7 初始位姿偏差为 $\left(0.52 \mathrm{~m}, 0.33 \mathrm{~m},-1.5 \mathrm{~m}, 15^{\circ}\right.$, $\left.15^{\circ}, 25^{\circ}\right)$ 的传统图像矩视觉伺服定位试验

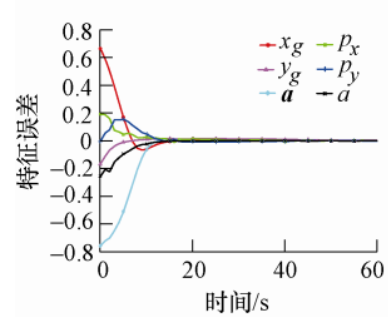

(a) 特征误差

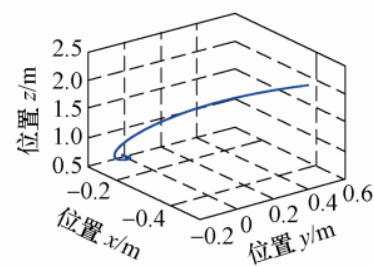

(c) 相机空间轨迹

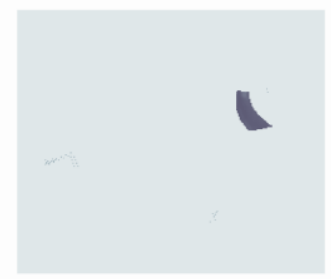

(e) 初始图像

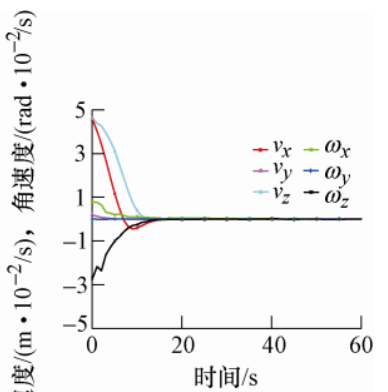

(b) 相机6自由度速度

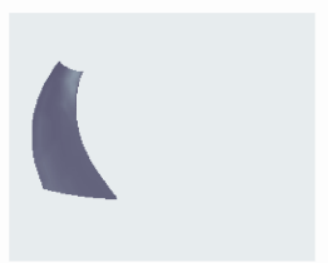

(d) 期望图像 I

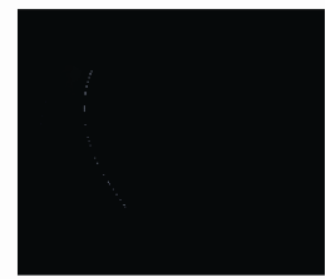

(f) 稳态时 $I-I^{*}$
图 8 初始位姿偏差为 $\left(0.52 \mathrm{~m}, 0.33 \mathrm{~m},-1.5 \mathrm{~m}, 15^{\circ}\right.$,

$\left.15^{\circ}, 25^{\circ}\right)$ 的深度矩视觉伺服定位试验

表 3 初始偏差较大的定位稳态误差

\begin{tabular}{|c|c|c|c|c|c|c|}
\hline & \multicolumn{6}{|c|}{ 位姿误差绝对值 } \\
\hline & $\Delta_{x} / \mathrm{mm}$ & $\Delta_{y} / \mathrm{mm}$ & $\Delta_{z} / \mathrm{mm}$ & $\theta_{x} /\left(^{\circ}\right)$ & $\theta_{y} /\left(^{\circ}\right)$ & $\theta_{z} /\left(^{\circ}\right)$ \\
\hline 传统矩 & - & - & - & - & - & - \\
\hline 视觉伺服 & & & & & & \\
\hline $\begin{array}{c}\text { 深度矩 } \\
\text { 视觉伺服 }\end{array}$ & 5.07 & 5.23 & 7.80 & 0.48 & 0.70 & 0.07 \\
\hline
\end{tabular}

图 7a 中, 传统图像矩视觉伺服在 $t=8 \mathrm{~s}$ 附近发 散; 图 7e 截取了 $0 \sim 8 \mathrm{~s} 5$ 个时刻图像, 目标快速 地偏离相机的视野范围(FOV)。深度矩视觉伺服在 初始偏差扩大的情况下，相较于初始偏差较小时 定位精度有所下降，但仍在 $t=20 \mathrm{~s}$ 附近收敛到稳 态, 呈现了较好的动态性能, 且保持了较优的空 间轨迹。

表 3 中, 定位误差偏大的主要原因是：图像特 征的初始偏差较大, 若控制律的比例因子 $\lambda$ 过大, 则控制超调, 导致目标脱离相机的视野范围, 收玫 失败。为了保证收玫性, 需要减小控制律的比例因 子, 保证在目标处于视野范围内, 直至逐渐收玫, 但带来精度下降的问题。

因此, 为了提高稳态时的定位精度, 在 $t=4 \mathrm{~s}$ 时, 增大比例因子。收敛过程如图 9 所示, 稳态误差见 表 4。通过调节控制器参数, 可以提高定位精度。 


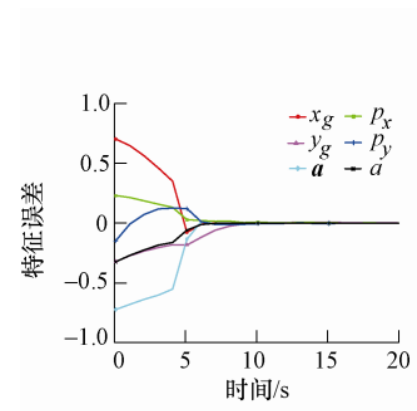

(a) 特征误差

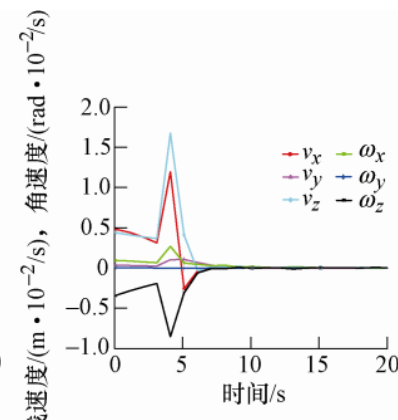

(b) 相机6自由度速度

图 9 初始位姿偏差为 $\left(0.52 \mathrm{~m}, 0.33 \mathrm{~m},-1.5 \mathrm{~m}, 15^{\circ}, 15^{\circ}\right.$, $25^{\circ}$ ), 调整控制器参数后的深度矩视觉伺服定位试验

表 4 调整控制器比例因子后定位稳态误差

\begin{tabular}{cccccccc}
\hline & \multicolumn{6}{c}{ 位姿误差绝对值 } \\
\cline { 2 - 6 } \cline { 5 - 6 } & $\Delta_{x} / \mathrm{mm}$ & $\Delta_{y} / \mathrm{mm}$ & $\Delta_{z} / \mathrm{mm}$ & $\theta_{x} /\left(^{\circ}\right)$ & $\theta_{y} /\left(^{\circ}\right)$ & $\theta_{z} /\left(^{\circ}\right)$ \\
\hline $\begin{array}{c}\text { 深度矩 } \\
\text { 视觉伺服 }\end{array}$ & 2.51 & 2.37 & 1.94 & 0.18 & 0.25 & 0.12 \\
\hline
\end{tabular}

两种视觉伺服方法均存在一定的定位误差, 无 法直接用于三维模型重建。文献[10]讨论了如何利 用 ICP(Iterative Closest Point 迭代最近点)配准算法 解决定位误差导致的精密建模问题。

\section{5 结论}

(1) 解算出传统矩特征的非参数化交互矩阵, 将传统矩视觉伺服控制方法用于曲面目标定位。在 初始偏差较小的情况下, 具有较高的定位精度, 收 敛速度慢。初始偏差较大时，无法收敛。

(2) 以深度矩作为图像特征的视觉伺服控制方 法可用于曲面定位。初始偏差较小时, 相对于传统 矩特征视觉伺服方法小幅提高定位精度，显著提高 收玫速度。初始偏差较大时, 仍然具有较好的动态 性能, 但是精度相对较低。在实际使用时, 可以考 虑在位姿偏差较小时, 重新调整控制器参数或者设 计自适应控制器, 获得更高的定位精度。

(3) 传统矩特征数视觉何服控制和深度矩视觉 伺服控制在定位过程均存在一定的误差，在重建过 程需要考虑如何处理该误差以获得更精确的三维模 型。为异形曲面测量过程的相机定位提供了一种具 备较强参考价值的思路。

\section{参 考 文 献}

[1] 史小强, 吴宝海, 张定华. 航空发动机叶片加工质量检 测技术发展现状与趋势[J]. 航空制造技术，2015， 481(12): 80-84.

SHI Xiaoqiang, WU Baohai, ZHANG Dinghua. Development tendency of inspecting technology for

aeroengine blade $[\mathrm{J}]$. Aeronautical Manufacturing Technology, 2015, 481(12): 80-84.

[2] 张宝峰. 航空发动机叶片测量现状及新方法分析 [J]. 科技创新导报，2016，13(20)：14-16.

ZHANG Baofeng. Current status of aeroengine blade measurement and analysis of new methods[J]. Science and Technology Innovation Herald, 2016, 13(20): 14-16.

[3] 谷天慧. 基于关节臂的发动机叶片测量方法研究 [D] 沈阳：沈阳航空航天大学, 2016.

GU Tianhui. Research on turbine blade measuring method based on articulated arm[D]. Shenyang: Shenyang Aerospace University, 2016.

[4] 李学哲, 石照耀, 陈洪芳, 等. 航空发动机叶片型面测 量技术研究现状与趋势 [J]. 北京工业大学学报, 2017, 43(4): 557-565.

LI Xuezhe, SHI Zhaoyao, CHEN Hongfang, et al. Current status and trends of aeroengine blade profile metrology $[\mathrm{J}]$. Journal of Beijing University of Technology, 2017, 43(4): 557-565.

[5] 㵶小军. 复杂零件自由曲面 CMM 测量关键技术研究 [D]. 西安: 西北工业大学, 2008.

LIN Xiaojun. Research on the key techniques of CMM measurement for free-form surface of complicated part[D]. Xi'an: Northwestern Polytechnical University, 2008.

[6] 郭成操. 叶片型面三坐标测量的自适应采样规划及算 法研究[J]. 工具技术，2010，44 (10): 99-104.

GUO Chengcao. Research on auto-adapted sample planning and algorithm in three-coordinate measurement of leaf blade profile[J]. Tool Engineering, 2010, 44 (10): 99-104.

[7] 于红英, 赵广亮, 刘文涛, 等. YP02 型叶片测量仪控 制系统的研制[J]. 制造技术与机床，2006(9): 73-75.

YU Hongying, ZHAO Guangliang, LIU Wentao, et al. Development of controlling system for blade measuring machine YP02[J]. Manufacturing Technology and Machine Tools, 2006(9): 73-75.

[8] 李进. 航空发动机叶片的型面质量测量方法对比 [J]. 中国新技术新产品, 2015(18): 6 .

LI Jin. Comparison of profile quality measurement methods for aeroengine blades[J]. Chinese New Technologies and Progucts, 2015(18): 6.

[9] MENG Zehui, QIN Hailong, CHEN Ziyue, et al. A two-stage optimized next-view planning framework for 3-d unknown environment exploration, and structural reconstruction[J]. IEEE Robotics and Automation Letters, 2017, 2(3): 1680-1687.

[10] KRIEGEL S, RINK C, BODENMULLER T, et al. Efficient next-best-scan planning for autonomous $3 \mathrm{D}$ surface reconstruction of unknown objects[J]. Journal of 
Real-Time Image Processing, 2015, 10(4): 611-631.

[11] VASQUEZ-GOMEZ J I, SUCAR L E, MURRIETA-CID R. View/state planning for three-dimensional object reconstruction under uncertainty[J]. Autonomous Robots, 2017, 41(1): 89-109.

[12] MONICA R, ALEOTTI J. Contour-based next-best view planning from point cloud segmentation of unknown objects[J]. Autonomous Robots, 2018， 42(2): 443-458.

[13] VASQUEZ-GOMEZ J I, SUCAR L E, MURRIETA-CID $\mathrm{R}$, et al. Volumetric next-best-view planning for 3D object reconstruction with positioning error[J]. International Journal of Advanced Robotic Systems, 2014， 11(159): 1-13.

[14] CHAUMETTE F. Potential problems of unstability and divergence in image-based and position-based visual servoing $[\mathrm{C}] / /$ European Control Association. Proceedings of European Control Conferences, August 31- September 3, 1999, Karlsruhe. Piscataway, New Jersey: IEEE, 1999: 4549-4554.

[15] TAMADATZE B, PIAT N L F, MARCHAND E. A direct visual servoing scheme for automatic nanopositioning[J]. IEEE/ASME Transactions on Mechatronics, 2012, 17(4): 728-736.

[16] COLLEWET X C, MARCHAND E, CHAUMETTE F. Visual servoing set free from image processing $[\mathrm{C}] / /$ Institute of Electrical and Electronics Engineers. Proceedings of International Conference on Robotics and Automation, May 19-23, 2008, University of Southern Californi, Pasadena, California. Piscataway, New Jersey: IEEE, 2008: 81-86.

[17] TEULIERE C, MARCHAND E. A dense and direct approach to visual servoing using depth maps[J]. IEEE Transactions on Robotics, 2014, 30(5): 1242-1249.

[18] CHAUMETTE F, HUTCHINSON S. Visual servo control, Part I: Basic approaches[J]. IEEE Robotics \& Automation Magazine, 2006, 13(4): 82-90.

[19] CHAUMETTE F. Image moments: A general and useful set of features for visual servoing[J]. IEEE Transactions on Robotics, 2004, 20(4): 713-723.

[20] TAHRI O , CHAUMETTE F. Point-based and region-based image moments for visual servoing of planar objects[J]. IEEE Transactions on Robotics, 2005, 21(6): 1116-1127.

[21] SAHU U K, PATRA D. Shape features for image-based servo-control using image moments[C]//Institute of Electrical and Electronics Engineers. Proceedings of Annual IEEE India Conference (INDICON), December 17-19, 2015, Jamia Millia Islamia University, Delhi. Piscataway, New Jersey: IEEE, 2015: 1-6.

[22] BAKTHAVATCHALAM M , CHAUMETTE F , MARCHAND E. Photometric moments: New promising candidates for visual servoing[C]//Institute of Electrical and Electronics Engineers. Karlsruhe Institute of Technology. Proceedings of International Conference on Robotics and Automation, May 6-10, 2013. Karlsruhe. Piscataway, New Jersey: IEEE, 2013: 5241-5246.

作者简介: 彭伟星, 男, 1994 年出生, 博士研究生。主要研究方向为机 器人自主三维建模视觉伺服控制。

E-mail:18711047309@163.com

王耀南(通信作者), 男, 1957 年出生, 博士, 教授, 博士研究生导师。 主要研究方向为智能控制理论与智能信息处理。

E-mail: yaonan@hnu.edu.cn 